Voix et Images

voixetimages

\title{
Du même au Même ou du fini à l'infini
}

\section{André Brochu}

Volume 34, numéro 2 (101), hiver 2009

Louise Dupré

URI : https://id.erudit.org/iderudit/029474ar

DOI : https://doi.org/10.7202/029474ar

Aller au sommaire du numéro

\section{Éditeur(s)}

Université du Québec à Montréal

\section{ISSN}

0318-9201 (imprimé)

1705-933X (numérique)

Découvrir la revue

Citer ce compte rendu

Brochu, A. (2009). Compte rendu de [Du même au Même ou du fini à l'infini].

Voix et Images, 34(2), 157-162. https://doi.org/10.7202/029474ar d'utilisation que vous pouvez consulter en ligne.

https://apropos.erudit.org/fr/usagers/politique-dutilisation/ 


\section{P O É S I E}

Du même au Même ou du fini à l'infini

$++$

ANDRÉ BROCHU

Université de Montréal

Le dernier recueil de Fernand Ouellette, Présence du large ${ }^{1}$, est paru après le somptueux triptyque intitulé L'inoubliable ${ }^{2}$, mais il a été écrit avant - du moins le premier jet ${ }^{3}$. L'auteur en a retardé la publication de façon à faire coïncider la sortie de son triple recueil avec le cinquantième anniversaire de son entrée en littérature, marquée par la parution de Ces anges de sang ${ }^{4}$. Je parlerai donc d'abord de Présence du large, chronologiquement premier, puis de l'étonnante floraison qui a suivi. L'auteur ayant accédé à une fécondité en regard de laquelle, heureusement, la qualité de ses écrits n'est pas en reste - qualité et quantité s'épaulent l'une l'autre -, il se pourrait que d'autres publications viennent s'ajouter sous peu à celles dont je fais état dans la présente chronique. Il n'empêche : 900 pages de poésie, et quelle! feront une matière plus que suffisante! Du reste, faute d'espace, je traiterai essentiellement du premier des trois tomes, qui donne une juste idée de l'ensemble.

Devant une production aussi soutenue, la question se pose: l'auteur est-il doué d'une prodigieuse imagination qui l'entraînerait, comme un Victor Hugo, sur tous les sites de la sphère poétique? Fait-il concurrence à l'univers ou, tout au moins, au dictionnaire, comme Balzac prétendait la faire à l'état civil?

Eh bien, non! Chaque poème semble nous ouvrir les portes d'une seule et même réalité, celle d'une pensée infiniment accueillante à elle-même, à son rapport à soi et à une relation verticale à l'infini. Sans doute, comme l'écrit le poète lui-même dans une présentation récurrente (I, II et III, p. 4 de couverture), y a-t-il enracinement «dans la matière du monde», mais l'«aventure unique» qui en découle est l'affaire du moi, ou, comme il le répète souvent, de l'intime. Car l'intime c'est TOUT, grâce à l'intensité de l'engagement du poète. Voilà très certainement une poésie fort

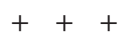

1 Fernand Ouellette, Présence du large, Montréal, l’Hexagone, coll. «L'appel des mots», 2008, 97 p. 2 Fernand Ouellette, L'inoubliable. Chronique I (Poèmes, 2003-2004), Montréal, l'Hexagone, coll. "L'appel des mots », 2005, 336 p.; Chronique II (Poèmes, 2003-2004), Montréal, l’Hexagone, coll. «L'appel des mots», 2006, 280 p.; Chronique III (Poèmes, 2003-2004), Montréal, l’Hexagone, coll. «L'appel des mots», 2007, 224 p. Désormais, ces ouvrages seront indiqués par le numéro de leur volume - I, II, III - , suivi du folio et placé entre parenthèses dans le texte. 3 Les renseignements biographiques proviennent de la documentation fournie par l'éditeur. 4 Fernand Ouellette, Ces anges de sang, Montréal, l’Hexagone, coll. «Les matinaux », 1955, 30 p. 
personnelle (ce qui ne veut pas dire confidentielle), à première vue peu soucieuse de l'autre, mais cette poésie travaille pour tous en ce qu'elle cherche inlassablement le secret de la vérité profonde de soi. Vérité inconcevable sans un rapport à ce que d'aucuns appellent l'être, et que d'autres appellent Dieu. Fernand Ouellette est croyant, mais il est aussi poète et, à ce titre, met de côté toute propagande religieuse. Il parle de l'être (ou encore de l'âme, sans fausse piété). Cet être, le croyant (extralittéraire) le vit en relation avec une transcendance. Le poète, lui, le rencontre plutôt dans les gestes et les tourments, les exaltations de chaque jour, l'immanence d'un vécu soumis aux aléas d'une existence promise à la mort. Le moindre sentiment le met en rapport avec la consistance du moi-monde, de ce qui fait sens. Or, voilà le maître mot.

Le poème intitulé "Le tour», qui est une sorte d'art poétique, dénonce «L'éteigneur de sens/Qui nous mure» (73). Le sens, en effet, est premier, c'est lui qui donne accès au «large", à la "présence » qu'évoque le titre du recueil. L'ennemi de l'humain s'en prend à ce qui élève et libère, grâce à «sa machinerie/Prochaine, insidieuse,/Trempée on ne sait/Dans quel marécage./Et le poison/Qui atteint le fond du cœur. » (73)

On touche ici à un aspect important de l'art de Ouellette, qui trouve des images à la fois simples et inattendues, très adroites dans leur apparente naïveté. L'« éteigneur» de sens qui nous «mure», qui d'un même geste coupe le feu vital et élève un cachot sans air et sans lumière, il est tout près de nous avec sa machinerie très articulée, laquelle pourrait évoquer la prolifération des formalismes et des structuralismes, ou des économismes, et en même temps la promiscuité de l'immonde venu du marécage et insinué, tel un poison, jusqu'au cœur. Le sec et l'humide sont également corrosifs, également contraires au mouvement vers l'ailleurs.

Un dualisme certain sous-tend cette thématique. Le combat de l'ombre et de la lumière - si cher à Hugo, par exemple - n'est pas loin. Chez Ouellette, la lumière est associée à l'enfance, et les derniers poèmes, dédiés à la petite Laurence, s'intitulent «Lumières du cœur». Le poète met en garde sa petite-fille contre «ce qui veut [lui] lier l'esprit,/[Lui] empierrer l'être» (81), bref contre ce qui éloigne de l'esprit d'enfance que caractérisent l'élan, le «besoin consumant/D'altitude» (86), ou encore la disposition du visage «À la tension éperdue de l'acquiescement./À l'ouverture vive, éclatante/Du sourire.» (79) Les thèmes les plus positifs de la sagesse occidentale et chrétienne sont réveillés, ravivés, viennent redire un essentiel dont on découvre, étonné, la subsistance. Ce n'est pas en refusant la tradition que Fernand Ouellette innove, mais en $s^{\prime} y$ ancrant et en faisant d'elle l'instrument d'une recherche du fondamental. C'est ainsi qu'il continue, cinquante ans après la parution de Ces anges de sang, de se rattacher à la grande problématique de l'enracinement qui est celle de sa génération, et de résister aux creux enchantements du nomadisme caractéristiques de la modernité. Car, à courtiser les «tumultes», à s'adonner aux «fuites éperdues»,

Qui n'en perdrait le cours des heures,

Le sens du battement intime,

Et n'en finirait par oublier

Qu'il est homme? (55) 
Voilà un humanisme tout simple, qui pourrait rappeler celui d'un Jean Royer, mais ce dernier est laïque et se donne des allures inspirées qu'on ne trouve pas ici. Chez Ouellette, le cœur («battement intime») comme instance naturelle, naturaliste du sens, s'impose avant tout.

Passons maintenant à d'autres beautés, en nombre effarant car elles s'additionnent au rythme de près d'un long poème par jour, pendant quinze mois (janvier 2003 - mars 2004). Vu l'abondance et la proximité de rédaction des textes, je traiterai comme un seul ensemble les trois recueils, sans chercher à rendre compte de la totalité.

On pourrait dire que les thèmes de L'inoubliable sont déjà réunis dans Présence $d u$ large, mais sous forme schématique, ce qui est conforme au dualisme qui les baigne. Or, voici que le poème double et même triple son espace énonciatif, que la parole acquiert non seulement une ampleur, mais une complexité qu'elle n'avait pas. Entre l'abîme redouté et le sommet visé, une dense végétation d'idées fait son apparition, et les images gagnent en polyvalence et en richesse. Le haut sans doute reste identifié au bleu, inaltérable d'un poème à l'autre, et le bas, quand il ne se colore pas d'origine ou d'enfance, se charge des affres boueuses, des tumultes de la condition humaine. Mais leur mise en rapport est beaucoup plus détaillée, et le bas, par ses aspects positifs, devient même le garant du haut: «Sans une attirance impérieuse/ Pour l'origine, nous perdons l'art même/De l'élévation, la direction de l'essor » (I, 116). L'origine (ou l'enfance, qui l'incarne) et le terme lumineux de l'élan, l'alpha et l'oméga de la vie, coïncident ou encore, se font voisins: "Tant que nous habitons dans les parages/Du natal, la mort continue de nous assiéger» (I, 235). Sans doute n'y a-t-il pas fusion des contraires, mais l'âpreté des conditions de l'existence demande à être patiemment réduite, pour que triomphe la familiarité «avec le vrai carrefour/Où se croisent les anges, les lumières » (I, 237).

Qui plus est, la temporalité interne du poème a changé. Il se veut maintenant "chronique», rend compte de l'instant même, tel que chaque jour le fonde à nouveaux frais:

\author{
EN ALLÉGRESSE \\ Aujourd'hui, \\ Sans briser son intensité, \\ Le verbe naissant laisse filer très haut, \\ En signe de promesse, \\ L'oiseau premier qui ose le vertical: \\ Éclair, glyphe à peine perceptible \\ Sur fond de bleu. (II, 9)
}

C'est «aujourd'hui» que se produit l'événement fondateur de la relation entre terre et ciel, de l'élan qui les unit. La personnification des acteurs abstraits ou du règne animal (verbe naissant, oiseau qui est éclair et glyphe) contribue à l'allure de 
chronique quotidienne où s'inscrit pleinement l'intemporel. Le concret et l'abstrait fusionnent, grâce au traitement fait à certains schèmes ou catégories sensibles tels le vertical ou le bleu (ce motif, rencontré plus haut, semble emprunté à Hölderlin ${ }^{5}$ et on connaît sa présence obsédante dans toute l'œuvre de Ouellette).

Les contraires se rapprochent aussi. La bienfaisante fixité de l'être ne s'oppose plus aussi nettement aux dangers de l'errance. Le poème intitulé «Nid», qui évoque d'abord un univers rassurant où «l'étoile se dépose au creux de la main" (I, 50), où la hauteur extrême se soumet à la mesure humaine, affirme bientôt l'excellence de l'excès: Car la démesure de la vision/Fait fuir la folie/Plus qu'elle ne la provoque. $(\mathrm{I}, 50)$

Sans doute la vision est-elle ce qui rapproche l'homme de l'infini, et elle ne nous éloigne pas de l'être; mais elle est une salutaire incitation au mouvement, puisque

\author{
Personne ne se veut vivant \\ Pour se cantonner dans les ornières, \\ [...] Car la limite, la pesanteur nous essoufflent \\ Beaucoup plus que l'envol (I, 50).
}

L'errance, qui est délire, procure dès lors la chance d'un chant qui délivre (I, 51). Le poète (c'est la conclusion de ce beau texte) voit trop souvent l'élan «qui se retourne en [lui],/Se cabre, se retire de l'allégresse,/S'évanouit/En perdant sa légèreté naissante » (I, 51). Il n'y a donc pas un enracinement bénéfique opposé à une errance sans but et sans profit, mais un appel de l'être qui rend possible l'inscription de l'infini dans l'action quotidienne.

Les significations nous sont données dans une formulation qui ne vise pas avant tout la clarté ou l'élégance (imaginons, par exemple, plutôt que les deux vers cités plus haut: Celui qui veut vivre/Ne se cantonne pas dans les ornières). La phrase, conforme au propos éthico-existentiel, se fait parfois lourde, difficile, ce qui exige du lecteur un premier effort de décryptage qui l'amènera à mieux s'interroger sur le sens du message. Comme l'écrit le poète, la parole "évite les consolations, les sentes/Trop facilement trouvées,/Trop bien tracées/qui ne conduisent que vers/Les précipices, les failles, les marais,/D'où rien ne peut s'envoler» (I, 86). La rhétorique enjôleuse, qui privilégie les sentes faciles, est agent de corruption et brise l'essor de l'âme.

Une touche de familiarité, voire de prosaïsme, un style de journal intime colorent parfois le poème, pour servir de tremplin à l'évocation de l'indicible:

Si nous pouvions soupçonner

À quel niveau de solitude nous sommes réduits,

Lorsque nous nous agrippons ainsi aux récifs,

5 Dans le poème intitulé "Apprentissage», Ouellette écrit ce vers complice: "En bleuité adorable, disait Hölderlin» (I, 68). 
Que nous nous dérobons aux pics,

Aux regards qui reviennent des hauteurs. (I, 56)

La généralisation à «nous» d'une situation individuelle confère à la phrase son côté sans-façon, ainsi que le verbe "soupçonner", lequel dramatise (ou romance) un état de conscience. Ce vocabulaire semi-intellectuel se dépasse vers les images, elles très "poétiques", qui disent la solitude. S'agripper aux récifs, se dérober aux pics, fuir les regards qui reviennent des hauteurs, voilà des actions apparemment similaires puisque coordonnées, et pourtant, seul l'imaginaire de l'auteur fonde leur synonymie. Affronter la noyade, refuser ce qui élève, écarter la présence des êtres supérieurs, tel est le sens d'images négatives dont la convergence ne se livre pas d'emblée.

Plus que bien d'autres, la poésie de Fernand Ouellette requiert le déchiffrement et la méditation. Non qu'elle soit énigmatique, au sens où elle appellerait une élucidation rationnelle, mais elle est imprégnée d'un symbolisme qui pointe moins vers les vérités éternelles que vers le mystère immédiat de l'existence. La patiente familiarisation avec ces symboles et motifs permet une lecture de plus en plus illuminante. Encore faut-il marcher dans les pas d'un homme qui ne pose ni au mystique ni au prophète - simplement un croyant pour qui l'univers est doté d'un sens et d'un ordre, d'origine divine. La conduite de vie, telle qu'évoquée de toutes les façons possibles à partir des conjonctures les plus diverses, fait l'unité de ce vaste traité du corps et de l'âme où le poète assume, en les traduisant intégralement dans la dimension sensible, les positions du philosophe et du théologien.

Mais qu'est-ce que cet «inoubliable» qui donne son titre à cette longue suite poétique?

Disons d'abord que le sens, mis au jour par le poème tel que le conçoit et le pratique Fernand Ouellette, se formule volontiers à travers des adjectifs substantivés, qui combinent le concret et l'abstrait («le paisible des grandes ailes» [I, 258]; «le prodigieux qui se propage» [I, 263] - je souligne). L'inoubliable en est un, et on peut s'étonner de sa relative rareté dans le texte, alors qu'il occupe la place d'honneur (le titre) dans le livre - contrairement au substantif bleu par exemple, présent partout mais non dans le titre. L'inoubliable, par là, apparaît comme le sens par excellence, vers lequel pointent les autres significations au delà de l'explicite.

Mais on peut tenter une réponse plus précise. Le poète, vers la fin du premier livre, évoque sa mémoire du ciel transparent, au sortir de l'abîme, mémoire

Jamais tout à fait engouffrée dans les âges.

Toujours présente, rayonnante,

[...] Et qui jamais n'a quitté le lieu

De l'inoubliable, où la gloire

Se métamorphose (I, 272). 
C'est l'inoubliable qui fonde la mémoire du vivant, et il est relatif à ce qui assure le passage de l'humain au divin, à cette "gloire» en laquelle la nature se fait lumière, devient participante au Verbe. Notons aussi que la mémoire, liée par définition à l'inoubliable, est un motif fréquent de cette poésie, et qu'elle nous reporte de préférence vers les instants radieux du passé, ce qui fait d'elle une faculté lumineuse. L'inoubliable, c'est donc ce que la mémoire des jours accumule en vue du triomphe sur les forces obscures et, en fin de compte, sur la mort, que la perspective chrétienne transforme en passage enviable. À la limite, l'inoubliable serait la part de Dieu en cette vie et se confondrait avec l'être même.

Notons, pour finir, que cette masse de textes que constitue le triptyque ne comporte pas de progression notable dans sa problématique, de sorte qu'il serait difficile d'établir, à partir d'elle, un choix de poèmes significatif. L'œuvre n'en explore pas moins les mille et une façons de circonscrire un Même essentiel, fini et infini ; un essentiel qui constitue ce pivot signifiant de l'inoubliable auquel se réfère chacun des textes. 